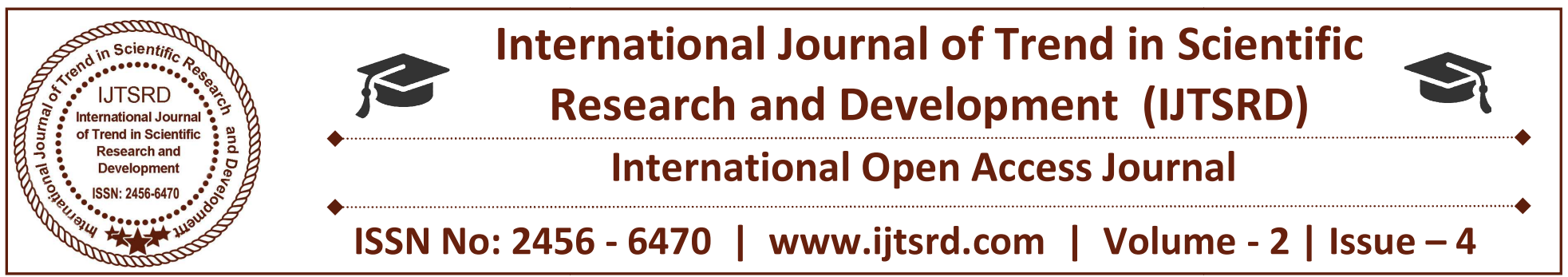

\title{
Impact of GST on Pharmaceuticals: An Overview
}

\author{
Kinjal Vaghasiya, Parvaiz Ahmad Parry, Neelam Somani ${ }^{1}$ \\ ${ }^{1}$ Assistant Professor \\ Department of Pharmaceutical Sciences, Mewar University, Chittorgarh, Rajasthan, India
}

\section{ABSTRACT}

GST also known as the goods and service tax is defined as the giant tax structure designed to support and enhances the economic growth of our country. More than 150 countries have implemented GST so far. GST is an indirect tax system which takes account expenditure done through sale, manufacture, and consumption of goods \& services at a national level which mainly involves companies, industries and services. The GST Rates for medicines were decided by the GST council. GST is levied under 5 different rates, namely NIL, $5 \%, 12 \%, 18 \%$ and $28 \%$ based on the HSN code of the item. GST is expecting to have a positive impact on the Indian Pharmaceutical Industries as it will decrease the manufacturing price since eight different taxes are levied in the pharmaceutical industries helps in easy going business. Beside some positive impact, there are some negative impacts also. A Drug Price Control is issued to make sure that the costs of vital medicines are fixed in such a way that they are affordable for everyone. According to the revised list issued by the National Pharmaceutical Pricing Authority( NPPA), prices of the majority drugs have come down, which includes a list of antibiotics and drugs for treating cancer, HIV, diabetes. GST also affects the Business Strategy of Pharmaceutical Companies.

Keywords: GST a giant tax structure, economic growth of country, Indirect tax system, GST rates, GST council, HSN code, Eight different tax, positive and negative impact, DPCO and essential medicines, NPPA and price control, business strategy

\section{INTRODUCTION}

GST: Goods \& Services Tax Law in India is a complete, multi-stage, destination-based tax that is levied on every charge addition.

In simple words, Goods and Service Tax is an indirect tax levied on the supply of goods and services. GST Law has replaced many indirect tax laws that earlier existed in India.

The tax came into consequence from July 1st, 2017 during the implementation of $101^{\text {st }}$ Amendment of the Constitution of India by the Prime Minister Narendra Modi .

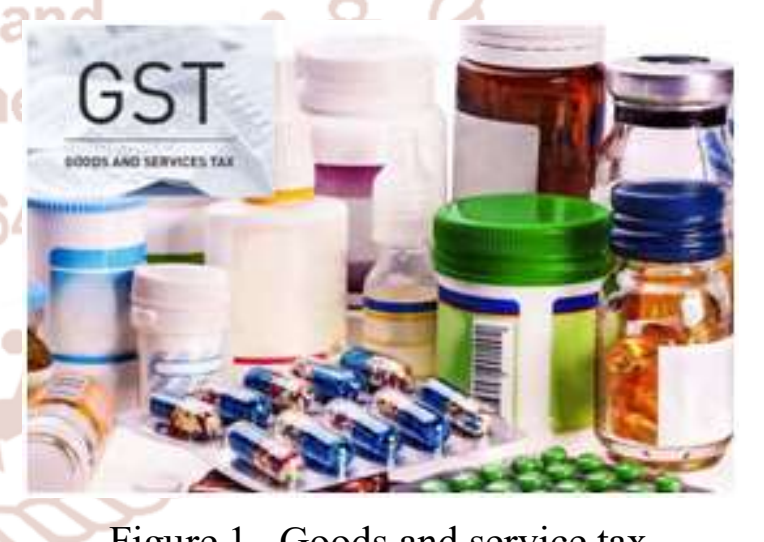

Figure 1. Goods and service tax

GST an meandering tax system which takes financial credit expenditure prepared through sale, manufacture, and consumption of goods \& services at a national level which mainly involves companies, industries and services area. The main idea behind it is to eliminate the tax levied of state and central government. It involves three stages

1) State GST (SGST) levied by state

2) Central GST (CGST) levied by centre 
3) Integrated GST (IGST) levied by central government on inter-state supply of goods and service. $^{3}$

\section{1) DRUG PRICE CONTROL ORDER (DPCO)}

Under the Essential Commodities Act, a Drug Price Control is issued to ensure that the costs of crucial medicines which are required in large amounts are set in such a way that they are reasonable for each person. The order has its roots in 1970 when the government realized the sick effects of the high profitability of medical drugs and the companies which made them. ${ }^{4,5}$

\section{2) CALCULATION OF RETAIL PRICE OF FORMULATION}

BEFORE GST: The retail price of a formulation shall be calculated by the Government in accordance with the following formula namely:

$$
\begin{aligned}
& \text { R.P. }=(\text { M.C. }+ \text { C.C. }+ \text { P.M. }+ \text { P.C. }) \times(1+ \\
& \text { MAPE } / 100)+ \text { ED. Where }
\end{aligned}
$$

- $\quad$ "R.P." means retail price;

- "M.C." means material price and includes the price of drugs and other pharmaceutical aids used including overages, if any, plus process loss thereon specified as a standard from time to time by notification in the Official Gazette in this behalf;

- "C.C." means conversion cost worked out in accordance with well-known procedures of costing and shall be fixed as a standard every year by notification in the Official Gazette in this behalf;

- $\quad$ "P.M." means cost of the packing material used in the packing of concerned formulation, including process loss, and shall be fixed as a standard every year by, notification in the Official Gazette in this behalf;

- "P.C." means packing charges worked out in accordance with well-known procedures of costing and shall be fixed as a standard every year by notification in the Official Gazette in this behalf;

- "MAPE" (Maximum A flowable Postmanufacturing Expenses) means all costs incurred by a manufacturer from the period of ex-factory cost to retailing and includes trade margin and margin for the manufacturer and it shall not exceed one hundred per cent for indigenously manufactured Scheduled formulations;

- "E.D." means excise duty: Provided that in the case of an imported formulation, the landed price shall form the basis for fixing its price along with such margin to cover selling and circulation expenses including interest and importer's profit which shall not go above fifty percent of the landed cost. ${ }^{6,7}$

\section{AFTER GST : $\quad$ GST $=($ MRP $) /(1+($ GST \%/100) $)$ \\ or $\operatorname{GST}=(\operatorname{MRP} \times 100) /(100+$ GST \% $)$}

The retail price of a formulation after GST shall be calculated by the Government in agreement by using this formula. ${ }^{8}$

\section{GST Rate for Medicines}

The GST Rates for medicines were decided by the GST Council in the meeting held on 3rd June, 2017. GST is levied in 5 different rates, specifically NIL, $5 \%, 12 \%, 18 \%$ and $28 \%$ based on the HSN code of the article. Medicines and pharmaceuticals are classified under 37 th chapter of the HSN Code.

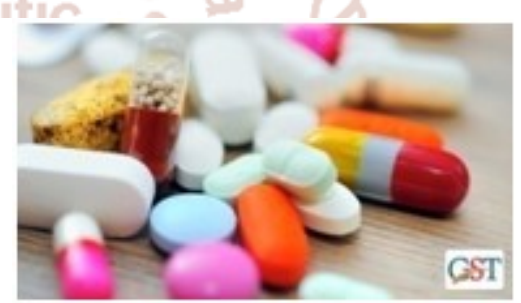

Figure2. GST rate for medicines

\section{1) NIL GST Rate Medicines}

The following types of medicines and pharmaceutical products are free from from GST:

- Human Blood and its components

- All types of contraceptives

\section{2) 5\% GST Rate}

Goods under the subsequent HSN categories are taxed at $5 \%$ GST rate:

- Animal or Human Blood Vaccines

- Diagnostic kits for detection of all types of hepatitis

- Desferrioxamine injection or deferiprone

- Cyclosporin

- Medicaments (including veterinary medicaments) used in bio-chemic systems and not bearing a brand name 
- Oral re-hydration salts

- Drugs or medicines including their salts and esters and diagnostic test kits

- Formulations manufactured from the bulk drugs

\section{3) $12 \%$ GST Rate}

The subsequent types of medicines and pharmaceutical goods are taxed at $12 \%$ GST rate:

- Organs for organo-therapeutic uses;

- extracts of glands or other organs or of their secretions for organo-therapeutic uses;

- heparin and its salts; other human or animal substances prepared for therapeutic or prophylactic uses, not elsewhere specified or included

- Animal blood prepared for curative, prophylactic or diagnostic uses;

- antisera and other blood fractions and customized immunological products, whether or not obtained by means of biotechnological processes;

- toxins, cultures of microorganisms (not including yeasts) and similar products.

- Medicaments consisting of two or more constituents which have been mixed together for therapeutic or prophylactic uses, not put up in measured doses or in forms or packings for retail sale, including Ayurvaedic, Unani, Siddha, homoeopathic or Bio-chemic systems medicaments.

- Medicaments consisting of mixed or unmixed products for curative or prophylactic uses, put up in measured doses (as well as those in the form of transdermal administration systems) or in forms or packings for retail sale, including Ayurvaedic, Unani, homoeopathic siddha or Biochemic systems medicaments, put up for retail sale.
- Wadding, gauze, bandages and similar goods (for example, dressings, adhesive plasters, poultices), impregnated or layered with pharmaceutical substances or put up in forms or packings for retail sale for medical, surgical, dental or veterinary purposes.

- Pharmaceutical goods such as Sterile surgical catgut, similar sterile suture materials (including sterile absorbable surgical or dental yarns) and sterile tissue adhesives for surgical cut closure; sterile laminaria and sterile laminaria tents; sterile absorbable surgical or dental haemostatics; sterile surgical or denatal adhesion barriers, whether or not absorbable, etc.,

- Waste pharmaceuticals.

\section{4) $18 \%$ GST Rate}

Nicotine polacrilex gum is the only medicine or pharmaceutical creation taxed at $18 \%$ GST rate. No pharmaceutical or medicines have been taxed at $28 \%$ GST. Hence, the maximum applicable GST rate for medicines is $18 \%{ }^{9,10}$

\section{HSN Code and GST Rate for Pharmaceuticals and Medicines}

HSN (Harmonized System of Nomenclature) is an 8digit code for identifying the appropriate rate of GST on different goods as per GST rules. If a company has earnings up to RS.1.5 Crore in the previous financial year then they need not mention the HSN code while supplying goods on invoices. If a company has earnings more than $1.5 \mathrm{Cr}$ but up to $5 \mathrm{Cr}$ then they need to mention the 2 digit HSN code while supplying goods on invoices. If turnover crosses $5 \mathrm{Cr}$ then they shall mention the 4 digit HSN code on invoices. ${ }^{11,12}$

\begin{tabular}{|l|l|l|l|l|}
\hline $\begin{array}{l}\text { HSN } \\
\text { codes }\end{array}$ & Description & $\%$ & $\begin{array}{l}\text { Implement } \\
\text { from }\end{array}$ & $\begin{array}{l}\text { Import } \\
\text { and } \\
\text { export }\end{array}$ \\
\hline 3001 & $\begin{array}{l}\text { Glands And Other Organs For Organo- } \\
\text { Therapeutic Uses; Extracts Of Glands Or } \\
\text { Other Organs Or Of Their Secretions For } \\
\text { Organo-Therapeutic Uses; Heparin And Its } \\
\text { Salts; Other Human Or Animal Substances } \\
\text { Prepared For Therapeutic Or Prophylactic } \\
\text { Uses, Not elsewhere Specified Or Included 13, 14, } \\
\text { 15 }\end{array}$ & $\begin{array}{l} \\
\text { Extracts of glands or other organ } \\
\text { Or of their secretions }\end{array}$ & $12 \% / 06 / 2017$ & \\
\hline 300120 & Liquid extracts of liver & $12 \%$ & & 30012010 \\
\hline
\end{tabular}


International Journal of Trend in Scientific Research and Development (IJTSRD) ISSN: 2456-6470

\begin{tabular}{|l|l|l|l|l|}
\hline & Liver extracts, dry & $12 \%$ & 30012020 \\
\hline & Snake venom & $12 \%$ & 30012030 \\
\hline & Other & $12 \%$ & & 30012090 \\
\hline 300190 & Other & $12 \%$ & & \\
\hline & Of human origin & $12 \%$ & & 30019010 \\
\hline & Heparin and its salt & $12 \%$ & 30019091 \\
\hline & Other & $12 \%$ & & 30019099 \\
\hline
\end{tabular}

Table: 1 Pharmaceutical comes under HSN 3001

\begin{tabular}{|c|c|c|c|c|}
\hline 3002 & $\begin{array}{l}\text { Animal Blood Products, Antisera And Other } \\
\text { Blood Fractions, Modified Immunological } \\
\text { Products, Toxins And }\end{array}$ & NIL & $28 / 06 / 2017$ & \\
\hline \multirow[t]{2}{*}{300210} & $\begin{array}{l}\text { Antisera and other blood fractions and } \\
\text { immunological products, whether or not modified } \\
\text { or obtained by means of biotechnological } \\
\text { processes: }\end{array}$ & NIL & & \\
\hline & Malaria diagnostic test kits & NIL & & 30021100 \\
\hline \multirow[t]{6}{*}{300212} & Antisera and other blood fraction & NIL & & \\
\hline & For diphtheria & NIL & & 30021210 \\
\hline & \begin{tabular}{l|l|l|} 
For tetanus & 0 \\
\end{tabular} & NIL & & 30021220 \\
\hline & For rabies & NIL & 3 & 30021230 \\
\hline & For snake venom thiternational Jour & NIL & & 30021240 \\
\hline & 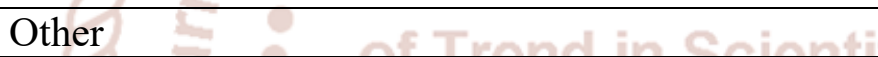 & NIL & (a) & 30021290 \\
\hline \multirow[t]{2}{*}{300213} & $\begin{array}{l}\text { Immunological products, unmixed, not put up in } \\
\text { measured does or in form or packaging for retail } \\
\text { sale }\end{array}$ & NIL & & \\
\hline & $\begin{array}{l}\text { Immunological products, unmixed, not put up in } \\
\text { measured does or in form or packaging for retail } \\
\text { sale }\end{array}$ & NIL & & 30021310 \\
\hline \multirow[t]{4}{*}{300214} & $\begin{array}{l}\text { Immunological products, mixed, not put up } \\
\text { measured doses or in form or packaging for retail } \\
\text { sale }\end{array}$ & & & \\
\hline & $\begin{array}{l}\text { Immunological products ,mixed, not put up } \\
\text { measured doses or in form or packaging for retail } \\
\text { sale }\end{array}$ & NIL & 8 & 30021410 \\
\hline & $\begin{array}{l}\text { Immunological products, put up measured doses } \\
\text { or in form or packaging for retail sale }\end{array}$ & NIL & & 30021500 \\
\hline & Other & NIL & & 30021900 \\
\hline \multirow[t]{11}{*}{300220} & Vaccines for human medicine & $5 \%$ & & \\
\hline & Single vaccines: & & & \\
\hline & For cholera and typhoid & $5 \%$ & & 30022011 \\
\hline & For hepatitis & $5 \%$ & & 30022012 \\
\hline & For tetanus & $5 \%$ & & 30022013 \\
\hline & For polio & $5 \%$ & & 30022014 \\
\hline & For tuberculosis & $5 \%$ & & 30022015 \\
\hline & For rabies & $5 \%$ & & 30022016 \\
\hline & For Japanese encephalitis & $5 \%$ & & 30022017 \\
\hline & For whooping cough(pertusis) & $5 \%$ & & 30022018 \\
\hline & Other & $5 \%$ & & 30022019 \\
\hline
\end{tabular}


International Journal of Trend in Scientific Research and Development (IJTSRD) ISSN: 2456-6470

\begin{tabular}{|c|c|c|c|}
\hline & Mixed vaccines: & & \\
\hline & For diphtheria, pertusis and tetanus(DPT) & $5 \%$ & 30022021 \\
\hline & For diphtheria and tetanus(DT) & $5 \%$ & 30022022 \\
\hline & For measles, mumps and Rubella(MMR) & $5 \%$ & 30022023 \\
\hline & $\begin{array}{l}\text { For typhoid - parathyroid(TAB) or typhoid- } \\
\text { parathyroid-cholera(TABC) }\end{array}$ & $5 \%$ & 30022024 \\
\hline & Other & $5 \%$ & 30022029 \\
\hline & Vaccines for veterinary medicine & $5 \%$ & 30023000 \\
\hline \multirow[t]{6}{*}{300290} & Other & NIL & \\
\hline & Human blood & NIL & 30029010 \\
\hline & $\begin{array}{l}\text { Animal blood prepared for therapeutic, } \\
\text { prophylactic, or diagnostic use }\end{array}$ & NIL & 30029020 \\
\hline & Probiotics & NIL & 30029030 \\
\hline & Toxins & NIL & 30029040 \\
\hline & $a \mathrm{CDON}$ & NIL & 30029090 \\
\hline
\end{tabular}

Table: 2 Pharmaceutical comes under HSN 3002

\begin{tabular}{|c|c|c|c|c|}
\hline \multirow[t]{10}{*}{3003} & $\begin{array}{l}\text { Medicaments consisting of two or more } \\
\text { constituents which have been mixed together for } \\
\text { therapeutic or prophylactic uses, not put up in } \\
\text { measured doses or in forms or packings for } \\
\text { retail sale, including Ayurvaedic, Unani, Siddha, } \\
\text { homoeopathic or Bio-chemic systems } \\
\text { medicaments. }{ }^{13,14,15} \text { Trend in Sient }\end{array}$ & 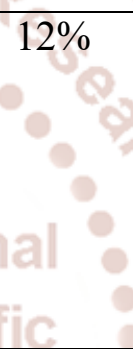 & $28 / 06 / 2017$ & \\
\hline & $\begin{array}{l}\text { Containing penicillins or derivatives thereof, } \\
\text { with a penicillanic acid S structure, or } \\
\text { streptomycins or their derivatives }\end{array}$ & $12 \%$ & & 30031000 \\
\hline & Other, containing antibiotics & $12 \%$ & $(6)$ & 30032000 \\
\hline & Containing Insulin & $12 \%$ & 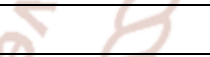 & 30033100 \\
\hline & \begin{tabular}{l|l} 
Other & SON: $2450=04 / 0$
\end{tabular} & $12 \%$ & & 30033900 \\
\hline & Containing ephedrine or its salts & $12 \%$ & & 30034100 \\
\hline & Containing pseudoephedrine or its salts & $12 \%$ & $\angle$ & 30034200 \\
\hline & Containing norephedrine or its salts & $12 \%$ & 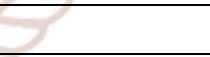 & 30034300 \\
\hline & 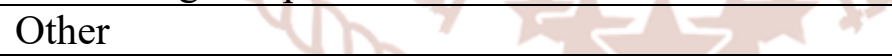 & $12 \%$ & & 30034900 \\
\hline & Other, containing anti-malarial active principles & $12 \%$ & & 30036000 \\
\hline \multirow[t]{10}{*}{300390} & Other & $12 \%$ & & \\
\hline & $\begin{array}{l}\text { Ayurvedic, Unani, siddha, Homoeopathic or bio- } \\
\text { chemic systems medicaments: }\end{array}$ & & & \\
\hline & Of ayurvedic system & $12 \%$ & & 30039011 \\
\hline & Of unani system & $12 \%$ & & 30039012 \\
\hline & Of siddha system & $12 \%$ & & 30039013 \\
\hline & Of homeopathic system & $12 \%$ & & 30039014 \\
\hline & Of bio-chemic system & $12 \%$ & & 30039015 \\
\hline & Menthol crystals & $12 \%$ & & 30039021 \\
\hline & Milk of magnesia & $12 \%$ & & 30039022 \\
\hline & $\begin{array}{l}\text { Bovine Albumin and drugs of animal origin, } \\
\text { Merbromine National Formulary XII } \\
\text { (Mercurochrome), Calcium Sennoside, } \\
\text { Anaesthetic agents used in human or veterinary } \\
\text { medicine or surgery, Aluminium hydroxide gel: }\end{array}$ & & & \\
\hline
\end{tabular}


International Journal of Trend in Scientific Research and Development (IJTSRD) ISSN: 2456-6470

\begin{tabular}{|l|l|l|l|l|}
\hline & Bovine albumin and drugs of animal origin & $12 \%$ & 30039031 \\
\hline & Merbromine national formulary XII & $12 \%$ & 30039032 \\
\hline & Calcium sennoside & $12 \%$ & 30039033 \\
\hline $\begin{array}{l}\text { Anaesthetics agents used in human or veterinary } \\
\text { medicine or surgery }\end{array}$ & $12 \%$ & 30039034 \\
\hline & Aluminium hydroxide gel & $12 \%$ & & 30039035 \\
\hline & Ketamine & $12 \%$ & 30039036 \\
\hline & Other & $12 \%$ & 30039090 \\
\hline
\end{tabular}

Table: 3 Pharmaceutical comes under HSN 3003

\begin{tabular}{|c|c|c|c|c|}
\hline 3004 & $\begin{array}{l}\text { Medicament consisting of mixed or unmixed } \\
\text { products for therapeutic or prophylactic uses, } \\
\text { put up in measured doses (including those in the } \\
\text { form of transdermal administration systems) or } \\
\text { in forms or packings for retail sale }{ }^{13,14.15}\end{array}$ & $12 \%$ & $28 / 06 / 2017$ & \\
\hline \multirow[t]{9}{*}{300410} & $\begin{array}{l}\text { Containing penicillin or derivatives thereof, } \\
\text { with a penicillanic acid structure, or } \\
\text { streptomycins or their derivatives }\end{array}$ & $12 \%$ & & \\
\hline & Penicillin & $12 \%$ & & 30041010 \\
\hline & Ampicillin & $12 \%$ & & 30041020 \\
\hline & Amoxicillin & $12 \%$ & $\mathrm{Q}$ & 30041030 \\
\hline & Becampicillin lntamention le & $12 \%$ & 2 & 30041040 \\
\hline & Cloxacillin & $12 \%$ & & 30041050 \\
\hline & Ampicillin and cloxacillin combination Scient & $12 \%$ & DIV & 30041060 \\
\hline & Streptomycin & $12 \%$ & 8 & 30041070 \\
\hline & Other rivesealcin allor & $12 \%$ & & 30041090 \\
\hline \multirow[t]{23}{*}{300420} & Other, containing antibiotics ayelonment & $12 \%$ & 3 & \\
\hline & Cephalosporins and their derivatives: & $12 \%$ & $(0)$ & \\
\hline & Cefazolin 0 & $12 \%$ & 2 & 30042011 \\
\hline & Cephalexin & $12 \%$ & 8 & 30042012 \\
\hline & Ciprofloxacin & $12 \%$ & & 30042013 \\
\hline & 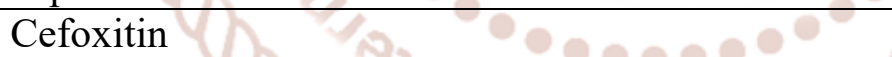 & $12 \%$ & & 30042014 \\
\hline & Other $\quad \mathrm{N}_{\mathrm{N}} \mathrm{Cu}$ & $12 \%$ & 7 & 30042019 \\
\hline & Sulphonamides and cotrimoxazole & $12 \%$ & & 30042020 \\
\hline & Fluoroquinolones: & $12 \%$ & & \\
\hline & Norfloxacin & $12 \%$ & & 30042031 \\
\hline & Nalidixic acid & $12 \%$ & & 30042032 \\
\hline & Ciprofloxacin & $12 \%$ & & 30042033 \\
\hline & Ofloxacin & $12 \%$ & & 30042034 \\
\hline & Other & $12 \%$ & & 30042039 \\
\hline & Tetracycline: & & & \\
\hline & Chlortetracycline & $12 \%$ & & 30042041 \\
\hline & Oxytetracycline & $12 \%$ & & 30042042 \\
\hline & Other & $12 \%$ & & 30042049 \\
\hline & Chloramphenicol & $12 \%$ & & 30042050 \\
\hline & Macrolides: & & & \\
\hline & Erythromycin & $12 \%$ & & 30042061 \\
\hline & Roxithromycin & $12 \%$ & & 30042062 \\
\hline & Clarithromycin & $12 \%$ & & 30042063 \\
\hline
\end{tabular}


International Journal of Trend in Scientific Research and Development (IJTSRD) ISSN: 2456-6470

\begin{tabular}{|c|c|c|c|c|}
\hline & Azithromycin & $12 \%$ & & 30042064 \\
\hline & Other & $12 \%$ & & 30042069 \\
\hline & Cefadroxil & $12 \%$ & & 30042070 \\
\hline & Other: & & & \\
\hline & Isoniazide & $12 \%$ & & 30042091 \\
\hline & Rifampicin & $12 \%$ & & 30042092 \\
\hline & Pyrazinamide & $12 \%$ & & 30042093 \\
\hline & Ethambutol & $12 \%$ & & 30042094 \\
\hline & Clindamycin & $12 \%$ & & 30042095 \\
\hline & Vancomycin & $12 \%$ & & 30042096 \\
\hline & Polymixin B and colistin & $12 \%$ & & 30042097 \\
\hline & Other & $12 \%$ & & 30042099 \\
\hline \multirow[t]{4}{*}{300431} & Containing insulin & $12 \%$ & & \\
\hline & Insulin injection & $12 \%$ & & 30043110 \\
\hline & 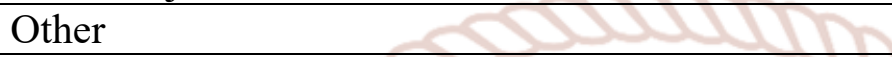 & $12 \%$ & & 30043190 \\
\hline & $\begin{array}{l}\text { Containing corticosteroid hormones, their } \\
\text { derivatives or structural analogues ClO }\end{array}$ & $12 \%$ & & 30043200 \\
\hline \multirow[t]{11}{*}{300439} & Other $\quad \mathrm{C}$ & $12 \%$ & & \\
\hline & $\begin{array}{l}\text { Pituitary hormones; Prednisolone; } \\
\text { Dexamethasone; Danazol; Other progestogen } \\
\text { and oestogen group hormones: }\end{array}$ & & & \\
\hline & Pituitary hormones & $12 \%$ & 8 & 30043911 \\
\hline & 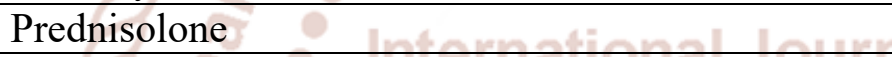 & $12 \%$ & 2 & 30043912 \\
\hline & Dexamethasone & $12 \%$ & & 30043913 \\
\hline & Danazole $\quad$ of Trend in Scient & $12 \%$ & DI & 30043914 \\
\hline & Other progestogen and oestogen group hormones & $12 \%$ & 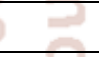 & 30043919 \\
\hline & Gonadotrophins and luteinising hormone: & & & \\
\hline & Gonadotrophins & $12 \%$ & 0 & 30043921 \\
\hline & Luteinizing hormone & $12 \%$ & 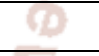 & 30043922 \\
\hline & Other & $12 \%$ & 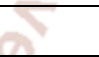 & 30043990 \\
\hline \multirow[t]{4}{*}{300440} & $\begin{array}{l}\text { Other, containing alkaloids or derivatives } \\
\text { thereof: }\end{array}$ & $12 \%$ & & \\
\hline & Containing ephedrine or its salts & $12 \%$ & $\angle$ & 30044100 \\
\hline & Containing pseudoephedrine (INN) or its salts & $12 \%$ & 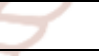 & 30044200 \\
\hline & Containing nor ephedrine or its salts & $12 \%$ & & 30044300 \\
\hline \multirow[t]{9}{*}{300449} & Other: $\quad$ : & $12 \%$ & & \\
\hline & Atropine and salts thereof & $12 \%$ & & 30044910 \\
\hline & Caffeine and salts thereof & $12 \%$ & & 30044920 \\
\hline & $\begin{array}{l}\text { Codeine and derivatives, with or without ephedrine } \\
\text { hydrochloride }\end{array}$ & $12 \%$ & & 30044930 \\
\hline & Ergot preparations, ergotamine and salts thereof & $12 \%$ & & 30044940 \\
\hline & Papavarine hydrochloride & $12 \%$ & & 30044950 \\
\hline & Bromohexin and salbutamol & $12 \%$ & & 30044960 \\
\hline & Theophylline and salts thereof & $12 \%$ & & 30044970 \\
\hline & Other & $12 \%$ & & 30044990 \\
\hline \multirow[t]{5}{*}{300450} & $\begin{array}{l}\text { Other, containing vitamins or other products of } \\
\text { heading 2936: }\end{array}$ & $12 \%$ & & \\
\hline & Heamatinics and Erythropoyetin preperations & $12 \%$ & & 30045010 \\
\hline & Preperations of minerals and their supplements & $12 \%$ & & 30045020 \\
\hline & Preparations of vitamins: & $12 \%$ & & \\
\hline & Of Vitamin A & $12 \%$ & & 30045031 \\
\hline
\end{tabular}


International Journal of Trend in Scientific Research and Development (IJTSRD) ISSN: 2456-6470

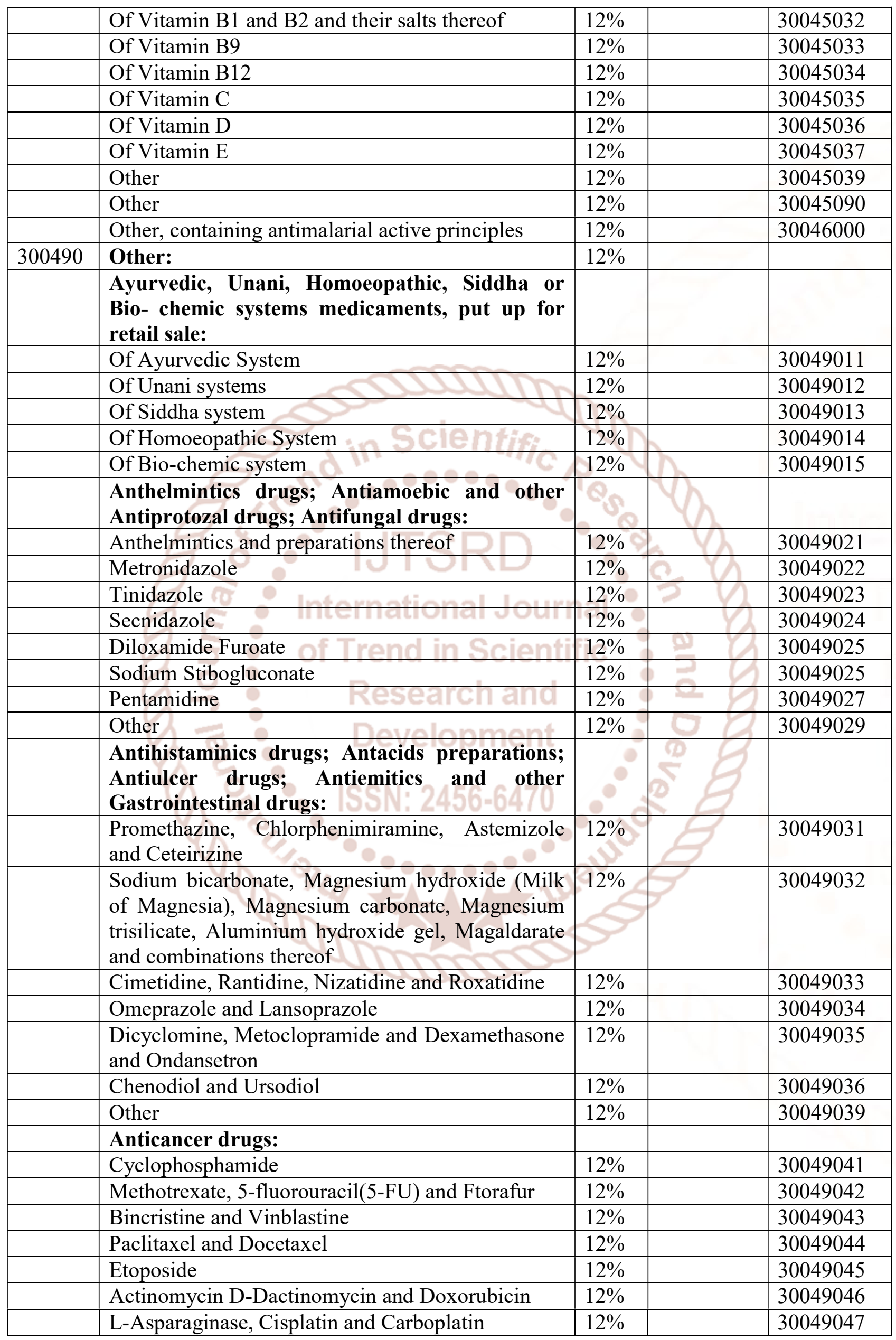


International Journal of Trend in Scientific Research and Development (IJTSRD) ISSN: 2456-6470

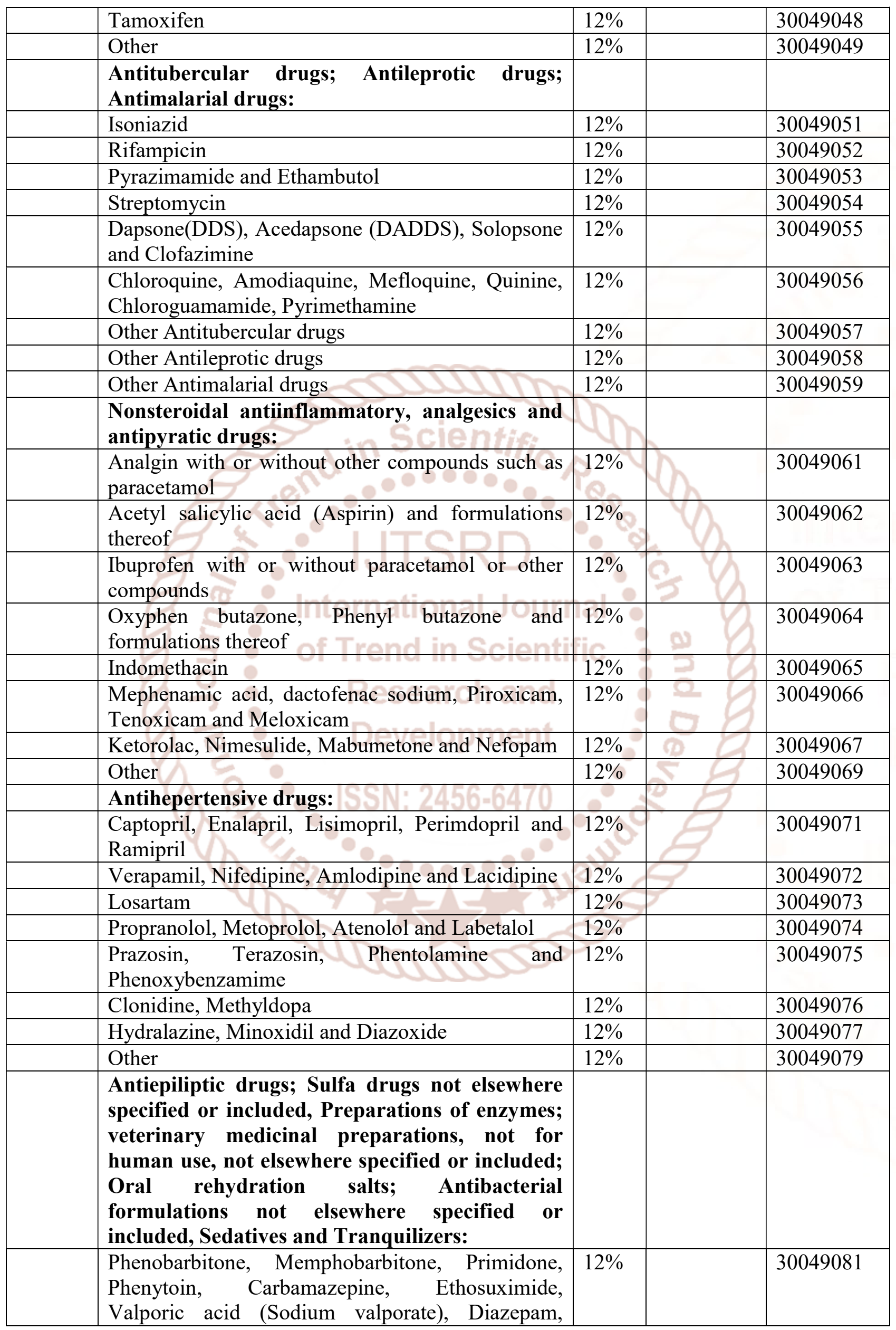


International Journal of Trend in Scientific Research and Development (IJTSRD) ISSN: 2456-6470

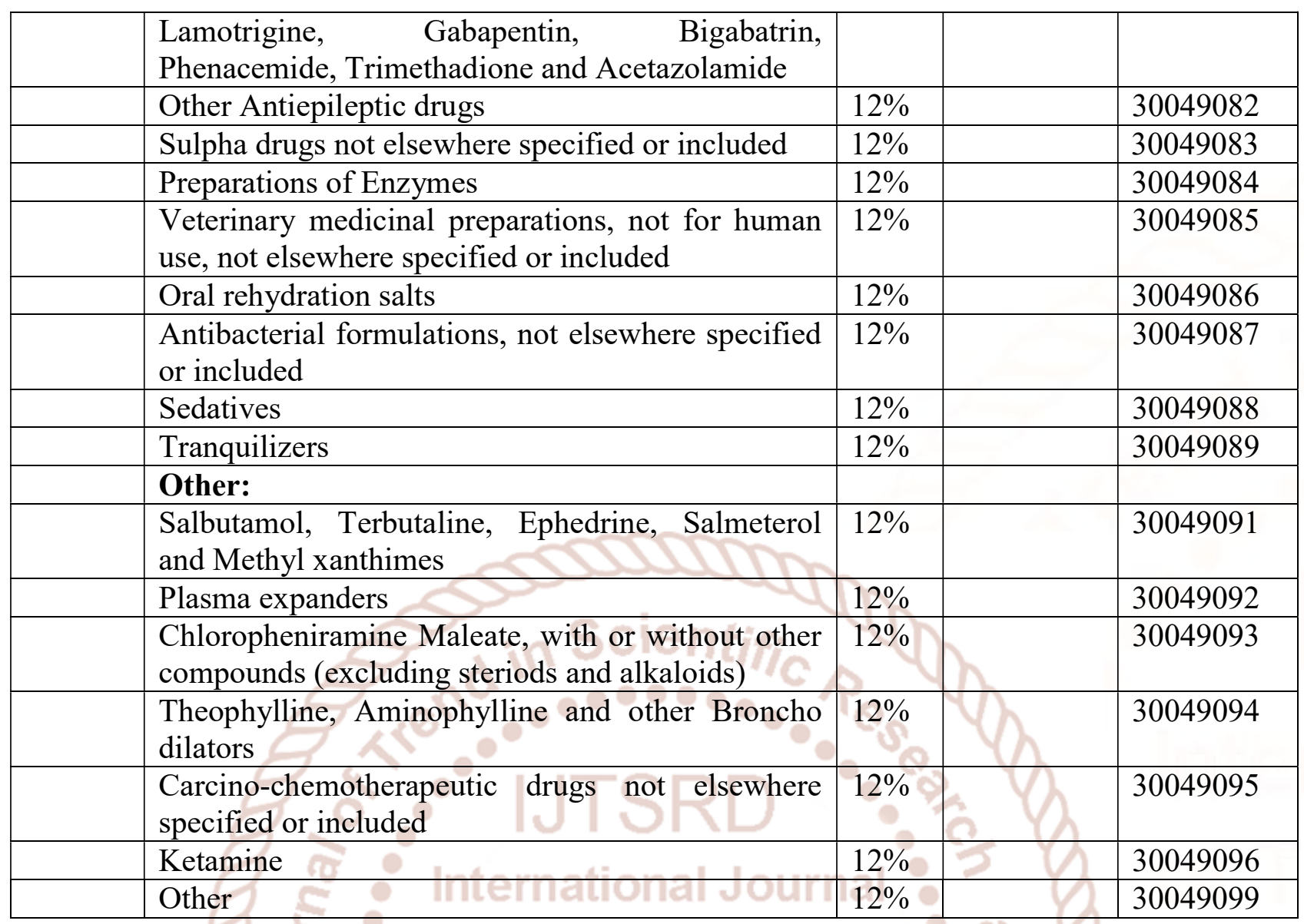

Table: 4 Pharmaceutical comes under HSN 3004

\begin{tabular}{|c|c|c|c|c|}
\hline 3005 & $\begin{array}{l}\text { wadding, gauze, bandages and similar article, } \\
\text { example, dressings, adhesive plasters, poultices), } \\
\text { with pharmaceutical substances or put up in for } \\
\text { packings for retail sale for medical, surgical, den } \\
\text { veterinary purposes } 13,14,15\end{array}$ & $12 \%$ & $28 / 06 / 2017$ & \\
\hline \multirow[t]{4}{*}{300510} & $\begin{array}{l}\text { adhesive dressings and other articles having an } \\
\text { adhesive layer: }\end{array}$ & $12 \%$ & & \\
\hline & adhesive guaze $\mathrm{n} \times \mathrm{y}$ & $12 \%$ & & 30051010 \\
\hline & 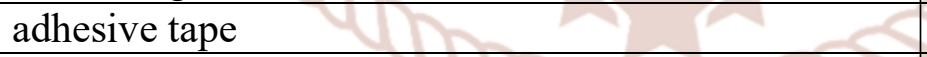 & $12 \%$ & & 30051020 \\
\hline & 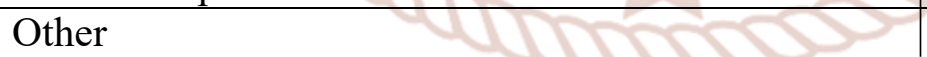 & $12 \%$ & & 30051090 \\
\hline \multirow[t]{9}{*}{300590} & other: & $12 \%$ & & \\
\hline & cotton wool, medicated & $12 \%$ & & 30059010 \\
\hline & poultice of kaolin & $12 \%$ & & 30059020 \\
\hline & Lint, medicated & $12 \%$ & & 30059030 \\
\hline & Bandages & $12 \%$ & & 30059040 \\
\hline & Burn therapy dressing soaked in protective gel & $12 \%$ & & 30059050 \\
\hline & Micro pores surgical tapes & $12 \%$ & & 30059060 \\
\hline & Corn removers and callous removers & $12 \%$ & & 30059070 \\
\hline & Other & $12 \%$ & & 30059090 \\
\hline
\end{tabular}

Table: 5 Pharmaceutical comes under HSN 3005 
International Journal of Trend in Scientific Research and Development (IJTSRD) ISSN: 2456-6470

\begin{tabular}{|c|c|c|c|c|}
\hline 3006 & $\begin{array}{l}\text { PHARMACEUTICAL GOODS SPECIFIED IN NOTE } \\
4 \text { TO THIS CHAPTER }\end{array}$ & $12 \%$ & $28 / 06 / 2017$ & \\
\hline \multirow[t]{7}{*}{300610} & $\begin{array}{l}\text { sterile surgical catgut, similar sterile suture materials } \\
\text { including sterile absorbable surgical or dental yarns) } \\
\text { nd sterile tissue adhesives for surgical wound closure; } \\
\text { terile laminaria and sterile laminaria tents; sterile } \\
\text { bsorbable surgical or dental }{ }^{13} 1415\end{array}$ & $12 \%$ & & \\
\hline & $\begin{array}{l}\text { sterile surgical catgut, similar sterile suture materials } \\
\text { (including sterile absorbable surgical or dental yarns) and } \\
\text { sterile tissue adhesives for surgical wound closure }\end{array}$ & $12 \%$ & & 30061010 \\
\hline & $\begin{array}{l}\text { sterile laminaria and sterile laminaria tents; sterile } \\
\text { absorbable surgical or dental haemostatics; sterile surgical } \\
\text { or dental adhesion barriers, whether or not absorbable }\end{array}$ & $12 \%$ & & 30061020 \\
\hline & blood grouping reagents & $12 \%$ & & 30062000 \\
\hline & $\begin{array}{l}\text { opacifying preprations for } \mathrm{x} \text {-ray examinations; diagnostic } \\
\text { reagents designed to be administered to the patient }\end{array}$ & $12 \%$ & & 30063000 \\
\hline & $\begin{array}{l}\text { dental cements and other dental fillings; bone } \\
\text { reconstruction cements }\end{array}$ & $12 \%$ & & 30064000 \\
\hline & first-aid boxes and kits & $12 \%$ & & 30065000 \\
\hline \multirow[t]{8}{*}{300660} & $\begin{array}{l}\text { chemical contraceptive preparations based on } \\
\text { hormones, } \\
\text { or on other products of heading } 2937 \text { or on spermicides: }\end{array}$ & NIL & & \\
\hline & based on hormones & NIL & & 30066010 \\
\hline & based on other products of heading 2937 lal Journall & NIL & & 30066020 \\
\hline & based on spermicides $T_{\text {und }}$ & NIL & 2 & 30066030 \\
\hline & $\begin{array}{l}\text { gel preparations designed to be used in human or veterinary } \\
\text { medicine as a lubricant for parts of the body for surgical } \\
\text { operations or physical examinations or as a coupling agent } \\
\text { between the body and medical instruments }\end{array}$ & $\begin{array}{l}\text { NIL } \\
0 \\
\end{array}$ & 5 & 30067000 \\
\hline & other: & & & \\
\hline & appliances identifiable for ostomy use 2456.6470 & NIL & & 30069100 \\
\hline & waste pharmaceuticals & 0 & & 30069200 \\
\hline
\end{tabular}

Table: 6 Pharmaceutical goods under HSN 3006.

\section{GST on India's Pharmaceutical Industry}

The $122^{\text {nd }}$ improvement Bill was passed as the Constitution's One Hundred and Twenty-Second Amendment Act, 2017. It will be affecting several industries, businesses, and companies at the same time.

The pharmaceutical industry occupies a unique case owing to the medicinal and pharmaceutical requirements it provides. If taken the share of GDP, it contributes to $5 \%$ of it. The Government of India with GST Council and its Chairman, Union Finance Minister of India - Arun Jaitley have introduced it so as to enhance FDI (Foreign Direct Investment). The alteration will bring about some changes in each individual industry. ${ }^{16}$
Impact of GST on Pharmaceutical Industry

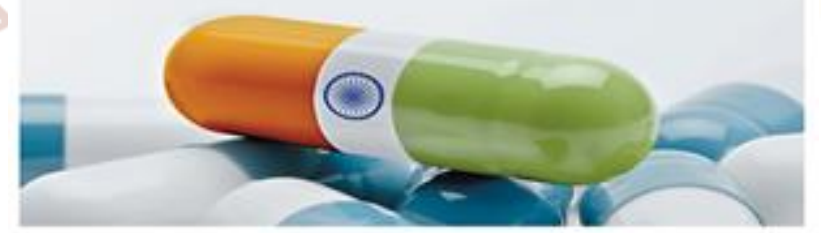

Figure 1. Impact of GST on pharmaceutical industries

Goods and Service Tax is expecting to have a encouraging impact on the Indian Pharmaceutical Industries as it will decrease the manufacturing cost since eight different taxes are levied in the pharmaceutical industries helps in easy going business. It will eradicate the cascading effect of multiple taxes applied on One Product. 
The Biggest reward for the Industries: conventional Cost and delivery Model will get replaced by supply chain efficiencies due to discontinuance of the Central Sales tax and interstate transactions between two dealers will become tax neutral. This will lead to a decrease in cost which can be added to margins and even consumers will get profit from it. This will provide absolute freedom to the companies to discover strategic supply chain and distribution channel. Additionally, it is uncertain till now that the healthcare services and lifesaving drugs will continue to be exempted or not under GST Regime. Under Current Indirect Taxation, these are exempted from Excise Duty and Custom Duty and a few states charge $5 \%$ taxes on Medicines.

The rate of GST should be kept at a relative level in the lowest slab for the pharmaceutical industries. Analysts specify the GST rate up to $12 \%$ to be neutral tax rate whereas no matter which above will have an inflationary effect on pricing. GST Council has finalized the tax rates of the goods in Nil, 5 percent, and 12 percent kind.

Beside some constructive impact, there are some unconstructive impacts also. As GST is relevant on phases of the supply chain, it will have a unhelpful impact on Free-drugs samples, Bonus/Discount Schemes, Inter-state stock transfer, etc. Pharmaceutical companies will experience better operational efficiency, condensed manufacturing \& transaction costs as well as better fulfillment. It will also promote warehousing strategy. As of now, companies kept their warehouses in different States to avoid Central Sales tax of different States. Now, they can combine warehouses at strategic locations as they will only have to pay Integrated GST (IGST) on interstate supplies of Goods and Services. ${ }^{17}$

\section{Positive Effects of GST On Economy of Pharmaceutical Industries}

GST eliminate the cascading effect of the tax which involved multiple taxes applied on only one Product. The costing and taxation system will be easy with only mountainous of three accounts. It will create a general market for every person with an equal chance towards development across various states.

Due to discontinue of the Central Sales Tax and Interstate transactions between two dealers the conventional Cost and Distribution Model will get replaced by supply chain efficiencies and tax will become impartial.

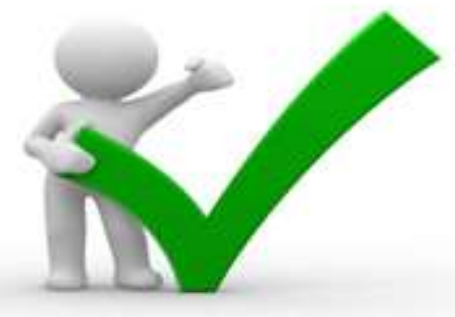

Figure 4. Positive effect of GST

1) Under GST, the life-saving vital drugs like the Oral re- hydration salts, Diagnostic Kits for detection of all types of hepatitis and various other life-saving injections ad drugs fall under the slab of $5 \%$.

2) Bonus/Discount Schemes, Free-drugs samples, Interstate stock transfer, etc are said to be costlier for firms for the reason that of its applicability of phases of the supply chain.

3) Pharmaceutical manufacturing generic/branded formulations/ dietary supplement companies who were suffer because of heavy excise duty will see a great profit due to condensed taxation and low industrialized cost for all firms.

4) The highest GST on bulk drugs is $18 \%$ and on formulations, it will be around $5 \%$ \& $12 \%$ at maximum. This means the formulators will be paying more tax through GST. Thus, they will be charged a smaller amount on formulations. It means the input credit will be accumulated which will be refunded itself.

5) Pharmaceutical companies and businessmen will find the lack of restrictions to explore strategic supply chain and distribution channel.

6) CENVAT credit is a credit balance in a bank account which can be refunded/ adjusted towards the central excise on the purchase or duty paid on a final product. The matter seems to finish for pharmaceutical industry due to GST charging single rate for goods and services. ${ }^{18}$

\section{Negative Effects of GST on Pharmaceutical Industries}

With helpful effect, there are a few unhelpful effects on the pharmaceutical industry:

1) Many Ayurvedic products are comes in the category of $12 \%$ to $15 \%$ as many of the ayurvedic products comes under cosmetic

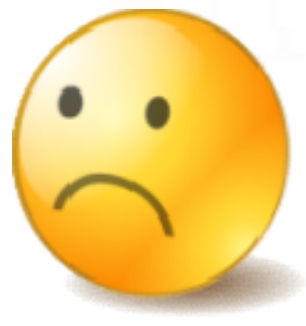

Page: 274 
range.

2) GST increase the indirect tax paid by pharmaceutical companies by $60 \%$ and MRP by 4 $\%$ Thus, leaving a Tax rate of $15 \%$ which is said to be $18 \%$ for diagnostics and reagents.

3) Other pharmaceutical drugs, medicines, Pharmaceutical products, and medical technology products are ranged flanked by $5 \%$ or $12 \%$ with frequently being a total tax (including VAT) of 11.5 to 12.5 percent to $18 \%$. $^{18}$

\section{REFERENCES:}

1) https://cleartax.in/s/gst-law-goods-and-services$\operatorname{tax}$

2) https://blog.saginfotech.com/gst-impact-onindian-pharmaceutical-industry

3) http://www.paxhealthcare.com/effect-of-gst-onpharma-industry/

4) https://www.gktoday.in/gk/drug-price-controlorders-dpco/

5) 5)Jain N.K., pharmaceutical jurisprudence, vallabh prakashan, $4^{\text {th }}$ revised edition 2015 , drug price control order 2013, page no.107-122.

6) http://www.nppaindia.nic.in/drug_price95/txt4.ht $\mathrm{ml}$

7) Dr. Agarwal S.P., Dr. Khanna Rajesh, Pharmaceutical Jurisprudence and ethics, Birla publication pvt.ltd., $5^{\text {th }}$ reprint edition 2015 , DPCO 1995, page no.193.

8) https://www.nilrisepharma.com/ptr-and-ptscalculator/

9) https://blog.saginfotech.com/gst-impact-medicineprices

10) https://www.indiafilings.com/learn/gst-ratemedicines/

11) https://en.wikipedia.org/wiki/Goods_and_Services _Tax_(India)

12) https://cleartax.in/s/drugs-pharmaceuticals-gstrate-hsn-code

13) https://www.indiafilings.com/learn/hsn-code-gstrate-pharmaceuticals-medicines/

14) https://www.seair.co.in/gst/hsn-codepharmaceutical-products.aspx

15) http://www.cybex.in/HS-Codes/PharmaceuticalProducts-Chapter-30.aspx

16) http://www.paxhealthcare.com/effect-of-gst-onpharma-industry/

17) https://blog.saginfotech.com/gst-impact-onindian-pharmaceutical-industry

18) http://www.paxhealthcare.com/effect-of-gst-onpharma-industry/ 